\title{
FUNNEL: TOWARDS COMFORTABLE EVENT PROCESSING
}

\author{
Burkhard D. Burow \\ Universität Hamburg, II. Instimu fiir Experimentalphysik \\ Luruper Chaussee 149, 2276/ Hamburg, Germamy
}

\begin{abstract}
The funnel software package has solved for the ZEUS collahoration the problem of Monte Carto few man-hours per day are suffecied by many HEP experiments. Thanks to extensive automation. a Carlo production. Other than specifyin resolve problems and to manage the entire ZEUS Monte from the chore of Montc Cur specifying the events to be produced, ZEUS physicists are thus freed for production are nearly cost production. As an additional benefit, the computing cycles required workstation and server computes, since they replace otherwise idle cycles on hundreds of unix are spread across a dozen sites around minimal interference for their regular users. The computers approximately one hundred dedicated computers and continually deliver the effective equivalent of
\end{abstract}

Funnel successfully demonstrates that generic independent tools can provide comfortable event processing. With an emphasis on automation and fault-tolerance. the tools manage all aspects of parallel processing. as well as dat queues. the execution and failures of the processing program. $\mathrm{HI}$ collaborations are presenty creang. archiving and remote transfer. The L3. HERMES and experience and to different extents, parts of Monte Carlo production systems. using the funnel exients, parts of the funnel software package.

The experience gained with funnel encourages the construction of EVPRO, a general purpose SPS or PVM for parallel processing. EVPRO would build on top of existing software: for example an application of any size could then easily on a dedicated farm of computers or using idle cycles processing. EVPRO aims to minimize applicatioy the comfon of automated, fault-tolerant event development costs can only be justified for the lapecific event processing software. whose high EVPRO with only the processing progra the largest of applications. A casual user may provide time application would tune EVPRO to its and the data to be processed. A more complex or realflow of event data. Making optima 10 its needs; for example. integrating custon hardware for the manage all aspects of the event processing the available computing resources. EVPRO would software triggers could use EVPRO, as could Monte Carlo production, event reconstruction and which can be expressed in terms of events any computing application, inside or outside of HEP,

In principle, event processing is a solved or even a trivial problem. Given an implementor. EVPRO
could provide the trivial solution in practice.

\section{Introduction}

The ZEUS experiment at DESY records of order $10^{7}$ events per year at the HERA electron-proton collider. The physics analyses require several times more Monte Carlo (MC) events than HERA events; on average $5 \times 10^{7} \mathrm{MC}$ events/year $\approx 10^{6} /$ week $\approx 100 /$ minute. Due to the large number of analyses, the required MC events are distributed across sumes one minute job requests per week. The MC simulation of an event typically consumes one minute of workstation CPU. Thus, ZEUS MC production effectively requires a. Presented in the plenary session 'F. Systems and Facilities' at Computing in High Energy Physics (CHEP95).
Rio de Janeiro, Brazil. 18-22 September 1995.

and by the BMBF under Contract Nr.056H H29l 
continual use of $10(0$ dedicated workstations. With an average $\mathrm{MC}$ event size of 60 Kbytes. the total production rate of $100 \mathrm{Kbyte} / \mathrm{second}$ can be carried by local area networks (LAN). though not generally on wide area networks (WAN). An aggregate production of about 60 Gbytes/week $=3$ Tbytes/year must be archived.

\section{I Resources for ZEUS MC Production}

There are approximately 500 physicists in the ZEUS collaboration, providing access to more than 500 workstations spread across sites in Europe. North America and Asia. Their otherwise idle CPU time is harnessed to effectively provide the 100 dedicated workstations required for MC production. At each site, the LAN connecting the 5 to 90 workstations allows MC production to use parallel processing. All the sites may be remotely controlled using ftp/telnet/rsh. The WAN, idle night bandwidth permitting. or Exabyte tapes sent by courier mail ${ }^{1}$ transport the produced events to the MC archive at DESY.

The above resources are managed by funnel to provide the ZEUS MC facility.

\subsection{The ZEUS Physicist's View of Fumnel}

Physicists submit jobs to funnel. Each job provides the input events, in terms of particle momentum vectors, to be sent through the MC simulation specified by the job. The requested detector and trigger simulation and event reconstruction may range from one of the standard ZEUS configurations through to a physicist's private development software.

Physicists receive from funnel for each job the archived output events, log files and information on any input events which crashed the simulation software.

ZEUS physicists are thus freed by funnel from the chore of MC production.?

\section{Global ZEUS MC Production}

\section{I Transferring Gbytes Around the World}

Each input job is sent over the network to one of the production sites for processing. To transfer data to a remote site, it is placed into a local transfer directory. This is an example of a daemon directon: It is monitored by a daemon process, which runs in the background and acts on every file appearing in the directory; logging the files, the actions and the exceptions handled. A successful action is noted by deleting the file or moving it elsewhere. Faults beyond the daemon's exception handling are e-mailed to the user. The action performed by the daemon is thus asynchronous, modular, automated and fault-tolerant. Once a process places a file in a daemon directory, the file requires no further actions nor exception handling from the process; the daemon effectively guarantees success.

Depending on the site, the output data is transferred to the MC archive by the farc daemon, using Exabyte tapes, or by the fmvbat daemon, using ftp.

\subsection{The Job Queue at Each Site}

To have an input job processed, it is placed into the local job queue directory. The job queue daemon ensures that for each job, the output data appears in the archive or, if the job fails in a manner beyond the daemon's exception handling abilities, e-mail notifies the funnel operator. For example, the daemon restarts a job aborted by a computer reboot and notifies the funnel operator if an input job is corrupt. 


\section{Comfortably Processing a Single Job}

In an ideal world, with infinite computer speed, unlimited disk space and bug-free programs, processing a single job is trivial. The processing program is simply run on the input

In the real worts.

In the real world, event processing is not trivial. A job may take days to complete and addition, others may want to use the neither time nor space are generally available. In or for some of the input events, the computer, the computer or disk or network may crash

The funnel tools can't provide the ideat wrogram may crash or enter an infinite loop. of the major tools show, they can provide its corld but, as the following outlines of some

\section{I Buffering Gbytes of Outpur}

The output data produced by a job is buffered on disk during processing. The buffering is performed automatically by the following two funnel tools. Once the job has completed. The getBUFFER links effectively place the buffered data into a transfer directory of section 2.1 .

getBUFFER returns an address anages free disk space. In response to a process' request, plus outstanding allocations on that disk with free space greater than the current request for use by the process. getBUFFER aut. Thus, the allocated space is guaranteed to be free cesses which have exited. getBUFFER dically reclaims allocations outstanding to proapplication. Thus one or more delete 'old' data; that's left up to the

The dogB utility places a process" may easily use multiple disks.

Unix VO to block the process until the getBUFFER getBUFFER area or areas. dogB uses gracefully blocked while the disks are full

Thanks to getBUFFER and are full, resuming once there is free space.

\subsection{Managed Event Processing and Parallel Event Processing}

The real world problems of job processing, those not related to disk space, are solved by making use of the natural unit of processing, the event. The input data consists of independent events, each of which is independently processed to produce an output event.

The solution keeps the simple original processing program, schematically shown in Figure la), but introduces the manager program as shown Figure $1 \mathrm{~b}$ ). The event is the manager is generic across different processing manipulating entire individual events, the event processing. For example, if using the idlo programs and can solve the problems of nizes when other users become active and idle cycles of a computer, the manager recoggram; resuming event processing once idlen terminates or suspends the processing pro-

Bugs are inevitable in large computer cycles are again available.

While bugs are eventually fixed, they are often such as the ZEUS simulation programs. processing. In analogy, HEP experiments don't usually hant and should thus not halt event line program occasionally crashes. Therefore, if the processing taking just because an onrent event and the stack trace are recorded, if the processing program crashes, the curing. As described in section 1.2, and jober, which then continues processinformation. The physicist can thus, a job's output data is accompanied by the crash sue the code's author(s). Similarly, if the proceselevance, if any, of a crash and can pur- 
a)
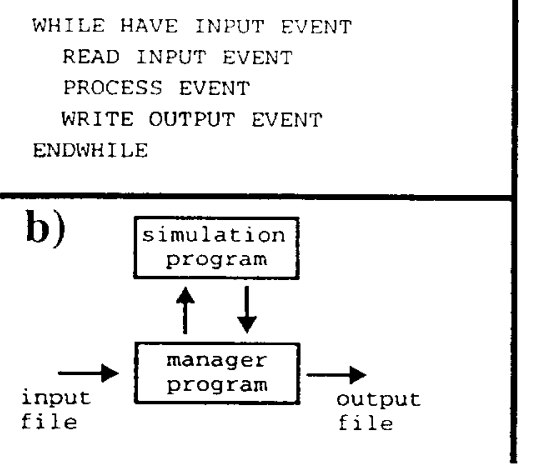

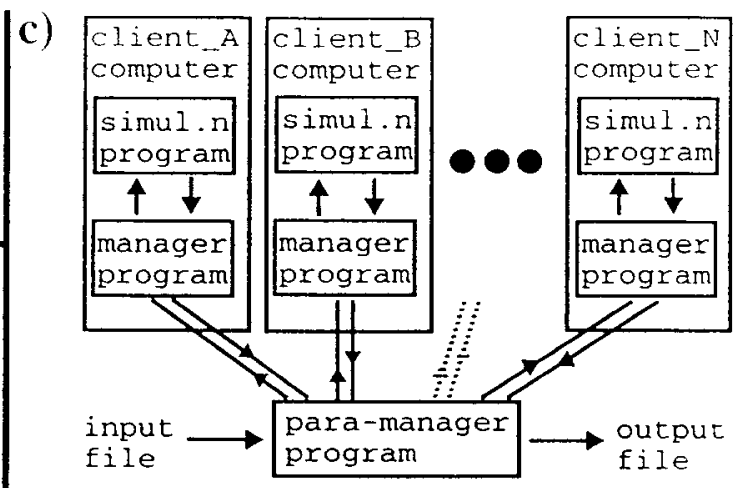

Figure 1 a) Pseudocode description of an evem processing program.

b) Event exchange with manager replaces direct access to input and output files.

c) Parallel event processing using the generic manager and para-manager tools.

manager e-mails the author(s), who can then examine the running loop with a debugger. The processing program is eventually forced to crash and is treated as described above

The independent events may obviously be distributed ${ }^{l}$ across many copies of the processing program. As illustrated in Figure lc), the para-manager program easily implements this trivial parallelism. As for the manager program, by manipulating entire events. the para-manager is generic across any processing program. Intrinsically simple, the paramanager provides various features such as the dynamic connection and disconnection of clients, allowing the use of idle cycles for processing.

\section{EVPRO for Comfortable Event Processing}

For event processing, be it MC production, a software trigger or event reconstruction. HEP is concerned with the input and output events, the processing software and any exceptions. HEP has no intrinsic interest in managing the computer resources passing the events through the processing software. As outlined in sections 2 and 3 , the funnel architecture demonstrates that generic, independent tools can manage the computing resources required for event processing. Going beyond ZEUS, funnel thus encourages the construction of EVPRO, a general purpose software package for event processing. EVPRO could benefit HEP tremendously, since event processing is currently managed manually and/or using application-specific software. This conference alone provides many examples ${ }^{3}$, in addition to funnel, of the HEP energy currently spent managing event processing; energy to be spared by EVPRO. Interest in EVPRO is corroborated by the L3, H1 and HERMES interest in funnel mentioned in the abstract. At present, EVPRO does not exist and seeks an implementor(s).

EVPRO would consist of event processing tools, containing some new software, and information helping the user determine the solution to the event processing problem. The solution may involve user, EVPRO and other software. The other software, which EVPRO would also heavily use, could include for example CPS or PVM for parallel processing. CERN's SHIFT utility for disk space management and NQS or LSF for job management. 
Passing a file of events through a simulation program demonstrates a simple use of
EVPRO. After relinking the simulation program to use the EVPRO manager's event read $\mathrm{ing}$ and writing routines and providing a routine identifying the input data event boundarles, the physicist would be freed of the details of the event processing, including parallel EVPRO would provide the tools requit exception handling. For many such jobs per day,

HEP software triggers using distributed funnel-like $M C$ production facility

EVPRO as illustrated in Figure 2a). The ager program which shares event memory withe for event $\mathrm{I} / \mathrm{O}$ is integrated into the man-

Outside of HEP, many applications with the trigger program.

which, the calculation time is greater thate similarly divisible into events or tasks, for EVPRO could provide comfortable master the data transfer time. As shown in Figure 2b),

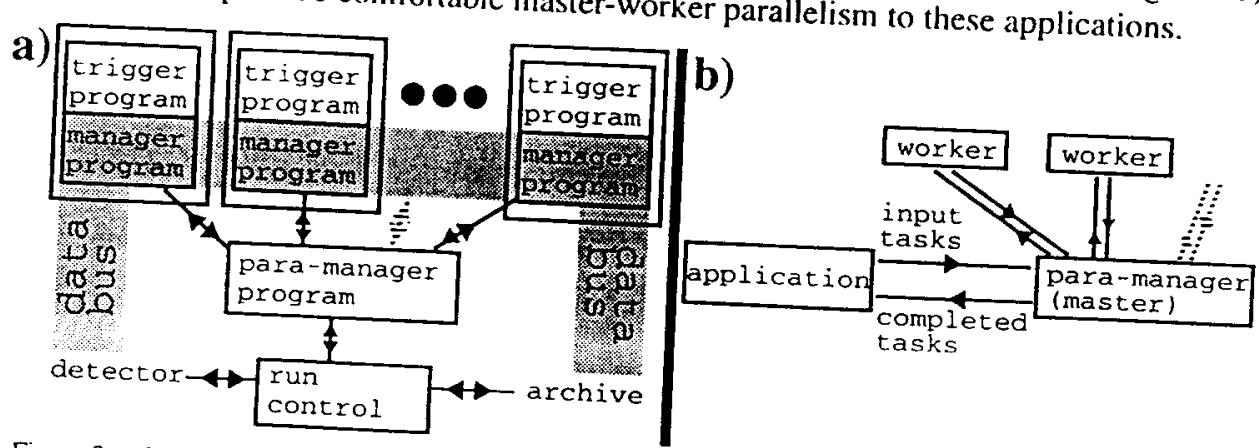

Figure 2 a) EVPRO for a HEP software trigger

b) EVPRO for generic master-worker parallelism.

\section{Summary}

Freed by funnel from the chore of MC event processing, ZEUS physicists have more time for physics. In addition, funnel provides inexpensive $\mathrm{MC}$ production using idle cycles

Implemented as EVPRO, funnel's idea of generic independention using idle cycles. should be portable to HEP and other applications. event processing, HEP should then have more ener. Freed by EVPRO from the chore of

\section{Acknowledgments}

Marc St-Laurent created the farc tape daemon. Wouter Verkerke created the software managing the global flow of jobs. ZEUS MC production and the funnel software owe much to A. Bruni, M. Botje and H. Wai, R. Yoshida, J. Butterworth, M. Kuze, D. Gilkinson, services and from the many ZEUS colleagues support comes from the DESY computing ware groups. Special thanks to ZEUS for all at the production sites and in the ZEUS soft-

\section{References}

1. The HEP precursors of techniques such as data transport by tape and event farming are described in the proceedings of previous CHEP conferences.

2. S.W. O'Neale, CHEP92, p.471, describes a similar service for OPAL.
3. M. Meesters. E. Anderson. A. Gellrich, R. Itoh. J Silva 
